\title{
Macrodipole Generated by Symmetry Breaking due to Phase Separation in Mixed Lipid Vesicles
}

\author{
Dietmar Porschke \\ Max Planck Insitut für biophysikalische Chemie, D-37077 Göttingen, Germany
}

Received: October 31, $1995^{\otimes}$

\begin{abstract}
A simple model calculation demonstrates that phase separation of components with different net charges in spherical mixed lipid vesicles leads to huge dipole moments. Experiments on mixed lipid vesicles composed of $50 \%$ dimyristoyl- $l$ - $\alpha$-phosphatidylcholine (DMPC) and $50 \%$ dimyristoylphophatidic acid (DMPA) demonstrate a large permanent dipole moment of about $8.1 \times 10^{-24} \mathrm{C} \mathrm{m}\left(\equiv 2.4 \times 10^{6} \mathrm{D}\right)$, which appears below a phase transition at about $30^{\circ} \mathrm{C}$. This phenomenon is characteristic of the mixed lipid system; it has not been observed for vesicles prepared from either DMPC or DMPA alone.
\end{abstract}

Some biological organisms can detect external electric fields with an extremely high sensitivity. ${ }^{1,2}$ This sensitivity raises questions about the sensing mechanism on the molecular level. Thermodynamics dictates that the sensitivity of any equilibrium toward the intensive parameter "electric field strength" is determined by the extensive "change of the electric moment": a transition $\mathrm{A} \Longrightarrow \mathrm{B}$ may be induced at low electric field strengths, if a high change of the dipole moment is associated with this transition. Thus, it is of interest to identify transitions with large changes of the dipole moment.

Lipid vesicles may be composed of many different lipids molecules, including both charged and uncharged residues. ${ }^{3,4}$ Under conditions without selective interactions between these molecules, the distribution is expected to be random. If the shape of the vesicles is assumed to be spherical, random distribution of molecules implies a virtually symmetric distribution of charges. The symmetry may be broken, when there are selective interactions between individual molecules. If the selective interactions are sufficiently strong, the components separate into different phases. It is instructive to model the case of complete phase separation under the assumption that the overall shape of the vesicles remains spherical. For simplicity it may be assumed that there is an equivalent number of charged and uncharged lipid molecules, which are segregated due to phase separation into the two halves of a sphere. This case of charged and uncharged lipids is selected, because it corresponds to the experiments described below. The nonsymmetric distribution of charges is quantified in terms of a dipole moment. Usually the dipole moment is defined for objects without net charge. The corresponding physical quantity for an object with a net charge must be referred to the center of diffusion, ${ }^{5-7}$ which corresponds in our case to the center of the sphere. We assume that after phase separation the charges are segregated on the surface of half of the sphere at an effective charge density $q_{\mathrm{d}}$ (cf. Figure 1 ). Using polar coordinates, the molecules in the surface area element $\mathrm{d} F=2 \pi r \cos (\varphi) r \mathrm{~d} \varphi$ contribute a dipole component $\mathrm{d} F \quad q_{\mathrm{d}} r \sin (\varphi)$. Integration provides a dipole moment $d_{z}$ in the direction of the $z$ axis (cf. Figure 1):

$$
d_{z}=q_{\mathrm{d}} 2 \pi r^{3} \int_{0}^{\pi / 2} \cos (\varphi) \sin (\varphi) \mathrm{d} \varphi=q_{\mathrm{d}} \pi r^{3}
$$

For vesicles with a radius $r=100 \mathrm{~nm}$ and a charge density $q_{\mathrm{d}}$ $\approx 6 \times 10^{-20} \mathrm{C} / \mathrm{nm}^{2}$ (estimated for a double layer with a lipid

\footnotetext{
${ }^{\otimes}$ Abstract published in Advance ACS Abstracts, December 15, 1995.
}

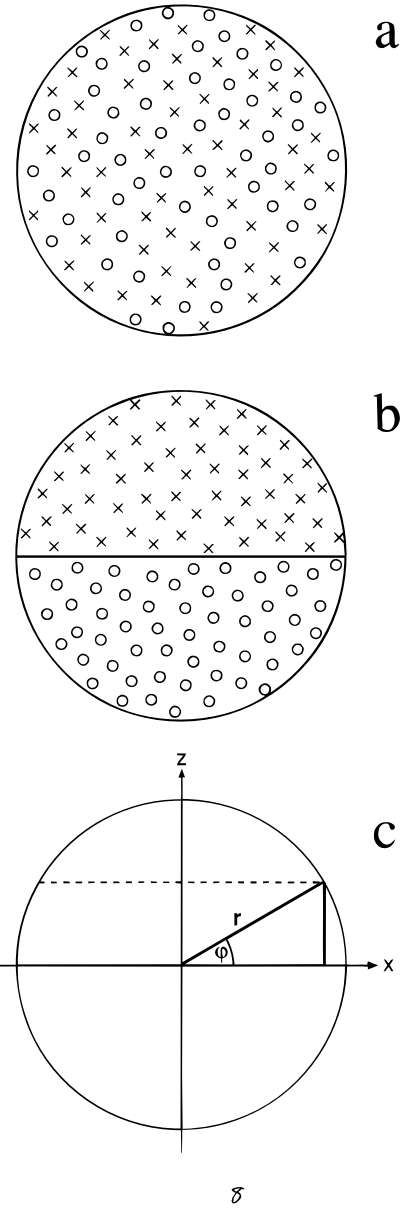

Figure 1. Scheme of a phase separation in a spherical vesicle: (a) symmetric distribution before phase separation, (b) limit case of complete phase separation, and (c) calculation of dipole moment under the assumption that charged lipids are segregated into the upper half of the sphere (cf. text).

packing density ${ }^{3,4}$ of $\sim 2$ molecules $/ \mathrm{nm}^{-2}$ in each layer and simply using the assumption, without reference to any particular model, of an effective charge density corresponding to $\sim 10 \%$ of the phosphate density), the expected dipole moment is $\sim 2$ $\times 10^{-22} \mathrm{C} \mathrm{m}$, corresponding to $\sim 6 \times 10^{7} \mathrm{D}$. Obviously this huge dipole moment represents an upper limit corresponding to a very high cooperativity of the phase separation; usually phase separations are not complete and the geometry of separated phases may not be equivalent to that of the simple 


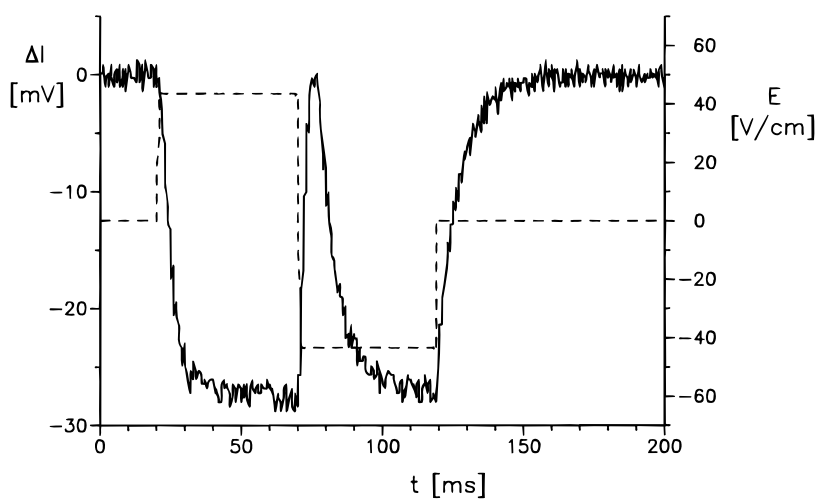

Figure 2. Change of the light scattering intensity $\Delta I$ induced by an electric field pulse $E$ with reversal of the field vector in a solution of vesicles containing 50\% DMPC and 50\% DMPA in a buffer with 1 $\mathrm{mM} \mathrm{NaCl}, 1 \mathrm{mM}$ Na cacodylate $\mathrm{pH} 7.0,200 \mu \mathrm{M}$ EDTA at $18{ }^{\circ} \mathrm{C}$; the concentration of DMPC and DMPA is $\approx 0.4 \mathrm{mM}$. The vesicles were prepared by the following procedure: equal molar amounts of DMPA (Sygena, Switzerland) and of DMPC (Sigma, Deisenhofen, Germany) were dissolved in benzene and then lyophilized; the buffer was quickly added under sonication to $2.5 \mathrm{mM}$ concentration of each lipid component; the preparation was tempered at $60{ }^{\circ} \mathrm{C}$ for $10 \mathrm{~min}$ and then passed through a sepharose $4 \mathrm{~B}$ column; the fraction with the highest vesicle concentration was used for the measurements.

model. Nevertheless, the model clearly demonstrates that huge dipole moments may be generated by phase separations.

For an experimental investigation, vesicles have been prepared from dimyristoyl- $l-\alpha$-phosphatidylcholine (DMPC), from dimyristoylphosphatidic acid (DMPA), and from mixtures of both lipids at various ratios. Vesicles of relatively small size distributions were isolated by gel chromatography. The vesicle preparations were then subjected to electric field pulses, and their response was analyzed by various optical techniques. The transients induced by rectangular field pulses with reversal of the field vector clearly demonstrate the existence of a permanent electric moment $^{8}$ (cf. Figure 2), when (1) the vesicle preparations contained equal molar amounts of DMPA and DMPC and (2) the temperature was below $\sim 30{ }^{\circ} \mathrm{C}$. These transients were observed by measurements of the light scattering intensity (with and without polarization of the light used), of the turbidity, of the birefringence and of the fluorescence detected linear dichroism using diphenylhexatriene as indicator. The stationary change of the light scattering intensity $\Delta I_{\mathrm{s}}$ induced by the field pulses approaches saturation already at low electric field strengths $E$ (cf. Figure 3). The dependence of $\Delta I_{\mathrm{s}}$ on $E$ is consistent with the orientation function for permanent dipoles and is not consistent with the orientation function for induced dipoles. ${ }^{8}$ According to a least-squares fit, the permanent dipole moment is $1.21 \times 10^{-23} \mathrm{C} \mathrm{m}$. After correction for the difference between the external applied electric field $E_{\mathrm{e}}$ and the internal directing electric field $E_{\mathrm{i}}$ using a simple sphere model (according to $E_{\mathrm{i}}=[3 \epsilon /(2 \epsilon+1)] E_{\mathrm{e}}$, where $\epsilon$ is the dielectric constant of the solvent ${ }^{10}$ ) the dipole moment is $8.1 \times 10^{-24} \mathrm{C} \mathrm{m}$, corresponding to $2.4 \times 10^{6} \mathrm{D}$.

The transients indicating permanent dipoles were not observed for vesicles prepared from either DMPC or DMPA alone but were also observed for mixed vesicles with mixing ratios from $25 \%$ DMPC/75\% DMPA to $75 \%$ DMPC/25\% DMPA. Measurements on vesicles with 50\% DMPC/50\% DMPA at different temperatures showed that the transients indicating permanent dipoles disappear at temperatures above $30{ }^{\circ} \mathrm{C}$ and reappear at temperatures below $30{ }^{\circ} \mathrm{C}$. This observation shows that the appearance of permanent dipoles is dependent on the separation of DMPA and DMPC phases. (The chain melting phase transitions of pure DMPA and DMPC bilayers ${ }^{3}$ are at 50 and $23{ }^{\circ} \mathrm{C}$, respectively.)

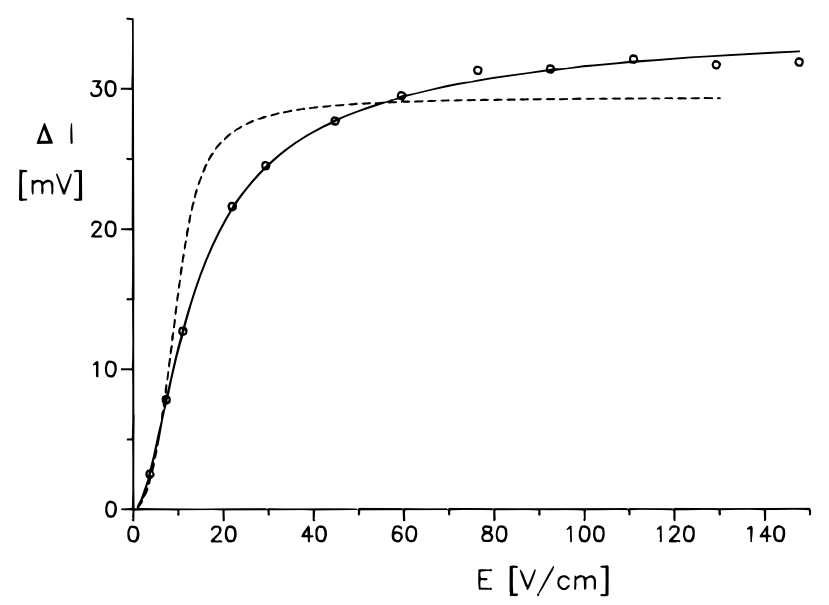

Figure 3. Stationary change of the light-scattering intensity $\Delta I_{\mathrm{s}}$ induced by pulses of the electric field strength $E$ in a vesicle solution containing $50 \%$ DMPC and 50\% DMPA in a buffer with $1 \mathrm{mM} \mathrm{NaCl}, 1 \mathrm{mM} \mathrm{Na}$ cacodylate, $\mathrm{pH} 7.0$, and $200 \mu \mathrm{M}$ EDTA at $18^{\circ} \mathrm{C}$. The continuous line represents a least-squares fit of the experimental data points according to the orientation function for dipoles with a permanent electric moment of $1.21 \times 10^{-23} \mathrm{C} \mathrm{m}$ and a limit value of the intensity change of 35 $\mathrm{mV}$; the dashed line represents a least-squares fit of the data by the orientation function for induced dipoles.

The vesicles used in the present investigation have been characterized by various techniques. Measurements of the dynamic light scattering showed particle diameters in the range around $200 \mathrm{~nm}$, approximately consistent with the electrooptical decay time constants. Electron microscopy revealed particles with a dominantly spherical shape of corresponding size. The nature of the optical signal induced by electric field pulses has to be studied in more detail. It is expected that there are fluctuations in the shape of the vesicles, which can be amplified by the electric field pulses. ${ }^{9}$ It is difficult to exclude the possibility that other than essentially spherical forms of lipid aggregates contribute to the electrooptical signal. Despite an uncertainty remaining in this respect, it is beyond any doubt that the transients indicating permanent dipole moments are dependent on the separation of phases. The process of phase separation should lead to large dipole moments for virtually any geometry of lipid aggregation. Obviously, these processes associated with huge changes of dipole moments are of considerable interest for field induced reactions at low electric field strengths. The separation of phases may be coupled to biological reactions either directly or indirectly, e.g., by changes of the protein distribution, which may lead to changes of their function.

Acknowledgment. The author is indebted to Dr. Derek Marsh for instructive comments, to Wolfgang Jahn for an analysis of the vesicles by electron microscopy, and to Prof. Manfred Kahlweit for generous access to his dynamic lightscattering facility.

\section{References and Notes}

(1) Kamjin, A. J. Science 1982, 218, 916-918.

(2) Heiligenberg, W. J. Exp. Biol. 1989, 146, 255-275.

(3) Cevc, G.; Marsh, D. Phospholipid Bilayers-Physical Principles and Models; Wiley Interscience: New York, 1987.

(4) Gennis, R. B. Biomembranes - Molecular Structure and Function; Springer: Berlin, 1989.

(5) Mysels, K. J. J. Chem. Phys. 1953, 21, 201-205.

(6) Porschke, D. Biophys. Chem. 1985, 22, 237-247.

(7) Antosiewicz, J.; Porschke, D. Biophys. Chem. 1989, 33, 19-30.

(8) Fredericq, E.; Houssier, C. Electric birefringence and electric dichroism; Clarendon Press: Oxford, 1973.

(9) Kummrow, M.; Helfrich, W. Phys. Rev. A 1991, 44, 8356-8360.

(10) Bottcher, C. J. F. Theory of Electric Polarization; Elsevier: Amsterdam, 1973; Vol. I.

JP953215H 